\title{
A broad view on overspill routing in optical networks: a real synthesis of packet and circuit switching?
}

\author{
Erik Van Breusegem ${ }^{\mathrm{a}, *}$, Jan Cheyns ${ }^{\mathrm{a}}$, Davy De Winter ${ }^{\mathrm{b}}$, Didier Colle ${ }^{\mathrm{a}}$, \\ Mario Pickavet ${ }^{\mathrm{a}}$, Piet Demeester ${ }^{\mathrm{a}}$, Joris Moreau ${ }^{\mathrm{b}}$ \\ ${ }^{a}$ Departnent of Information Technology (INTEC), Ghent Untversity-LMEC, Sint-Pietershieuwstraat 4l, B-9000 Gent, Belgikm \\ 'Department INWE, Hogeschool Gent, J. Klwyskensstraat 2, B-9000 Gent, Belgiwn
}

Received 1 July 2004; received in revised form 30 September 2004; accepted 4 October 2004

Available online 18 November 2004

\begin{abstract}
The emerging wavelength switched networks reduce the strain on packet forwarding. Unfortunately, that solution is not really efficient on a bandwidth level, and is not ideally suited for bursty traffic. Packet switched solutions, whether electronic or optical, can use statistical multiplexing to cope with bursty traffic and yield better bandwidth efficiency. We present a novel network concept that can combine these two worlds, withholding their advantages. We introduce this Overspill Routing In Optical Networks (ORION), and discuss several aspects of it: the overall architecture and network concept, node design and implementation, and evaluation at network level as well as node level.
\end{abstract}

02004 Elsevier B.V. All rights reserved.

Keywords: Circuit switching; Optical switching; Packet switching; Wavelength division multiplexing

\section{Introduction}

In the past optical networks opened up a vast amount of bandwidth, with the breakthrough of optical fiber and all-optical amplification. A second boom in bandwidth capacity came with the development of (Dense) Wavelength Division Multiplexing, \footnotetext{
60 .

* Corresponding author. Tel.: +3292649965; fax: +32926499

E-mail address: Erik, VanBreusegem@intec.ugent.be (E. Van Breusegem).
}

(D)WDM. As a consequence, pure bandwidth availability is no longer the most important cost determining factor on the technological side. Originally, optical connections were used as high capacity pointto-point interconnections. Data was converted to the electronic domain every hop, and processed at an electronic router. This resulted in highly efficient sharing of the transmission resources. At the same time, however, most of the traffic handled in any router is transit traffic, i.e. still has to travel one or more hops to reach the exit point of the network. $70 \%$ of all traffic being transit is not an exception [1], which is of course 
mainly due to the low meshing degree of backbone networks. Given the current trend that optical transmission capacity grows faster [2] than electronic processing capability, the electronics will become a major bottleneck. Consequently, several solutions were proposed to reduce the strain on the electronic layer. The most important one certainly is the concept of wavelength paths (or lightpaths). In this technology a wavelength passes transparently through an optical crossconnect (OXC), without the IP router inspecting the carried data. The drawback of this approach is that a wavelength path cannot be re-used by intermediate nodes when it is not used at full capacity. This can result in an increased amount of wavelengths needed to fulfill a set of demands. A logical next step in this evolution are Automatically Switched Optical Networks (ASONs), where the wavelength paths are set up by the control plane, without (explicit) intervention of the network operator. This allows adapting to traffic variations on a medium to large time scale (several minutes to even hours or days). However, these solutions inherently remain wavelength switched. Although the flexibility is considerably increased, the granularity, in terms of bandwidth and time scale, is still coarse.

In this paper we describe a recently proposed switch and network architecture proposal, called Overspill Routing In Optical Networks (ORION), that succeeds in using the full benefits of the ASON, without sacrificing the high statistical multiplexing gains from the earlier point to point WDM optical networks. In contrast to earlier papers, we will look at ORION from a broader perspective, integrating existing results from recent and earlier work. The alloptical extensions were previously unpublished.

\section{Related work}

Although ORION as a hybrid is quite unique in its design and concept, several other proposals try to achieve similar objectives, through some kind of intermediate form of simple point to point WDM and wavelength switched networks. Since we haven't introduced ORION at this point, we will come back to these during the explanation and highlight some important differences with ORION when appropriate. The closest resemblance ORION has with any other proposals certainly is the polarization based concept described in $[3,4]$, as well as the light-trail and lightbus concepts [5], and [6]. Others may seem similar, like $[7,8]$, or [9], but during our discussion we will show that they are actually, in our view, an extreme form of more classical switching paradigms. The overspill mechanism we will explain is sometimes also likened to deflection routing [10] or the lighttree [11] concept, but we will also illustrate that it is fundamentally different. Finally, the CHEETAH [12] concept comes to mind as related. This is also a true hybrid, but is actually a form of a combined architecture, with two physically distinct networks in parallel (see also Section 7 for more details).

The remainder of the paper is organized as follows. First, we introduce the ORION concept from a network level. We continue by describing an ORION node in a very generic way. The node operation relies on the use of a special marker for packets, which is why we continue by suggesting a few ways for actual implementation of this marker. Then, we show a case study evaluation of ORION performance in a pan-European network from a dimensioning point of view for the network. This is followed by a simple case study at node level, where simulation can show the finer behavior of the overspill mechanism. A conclusion summarizes the content and indicates some other points of interest, already investigated (but not explained here) or still in the pipeline. Throughout the paper we will usually explain ORION using an IP over WDM setting, but it is important to note that the concept is not restricted to it. Other options may be to implement it in e.g. IP/MPLS/WDM, Gigabit Ethernet over WDM, or ATM/WDM.

\section{Architectural concept}

We will begin by explaining how ORION works at a network level. First, we start from a wavelength switched WDM network, where some wavelength paths are established. In a normal wavelength switched network a lightpath passes some node transparently, i.e. the node cannot access the data in the passing wavelength. In the example of Fig. 1 node $D$ has no access to the IP packets travelling in wavelength $\lambda_{1}$. Only wavelengths starting or terminating in that node can be accessed. Suppose we have the connections $A-a-b-c-d-D$ on $\lambda_{2}, B-b-c-d-e-E$ on $\lambda_{3}$ and $A-a-b-c-d-e-E$ on $\lambda_{1}$, established as indicated on 


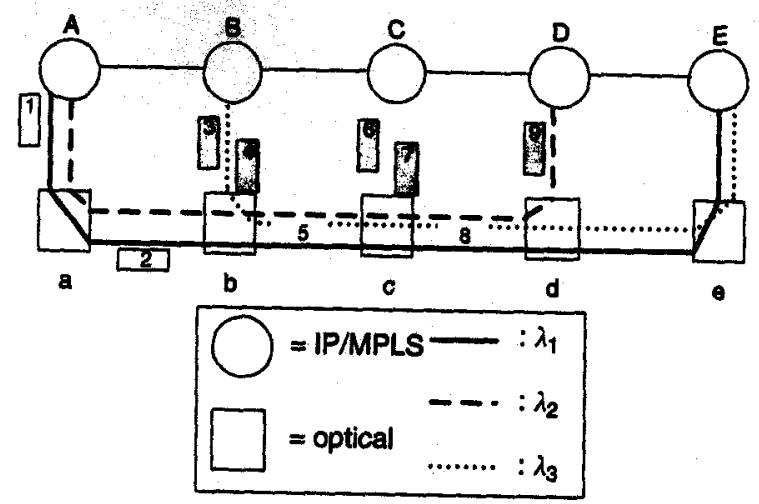

Fig. 1. A simple ORION network scenario.

Fig. 1. The basic problem with wavelength switching occurs in the following scenario: when the capacity of the lightpath $\mathrm{AD}$ is fully used, and there is some temporary overload for this connection, the wavelength $\lambda_{1}$ cannot be used to carry some of its traffic, as it is dedicated for connection AE. It is, however, perfectly possible that the lightpath $A E$ (on $\lambda_{1}$ ) is only carrying a low traffic load, so that in fact at some moments in time it is empty (as mentioned, we focus on IP directly over WDM here). Thus, while connection $\mathrm{AD}$ has a capacity shortage, $\mathrm{AE}$ has capacity in abundance.

While ordinary wavelength switched WDM networks cannot solve this type of situation instantly (since it requires a reconfiguration of the network), ORION enables the network to use this capacity on $\lambda_{1}$ (normally dedicated to the connection AE) for the connection $\mathrm{AD}$ by switching to the so-called overspill mode, which essentially means operating the network as if it is a point to point WDM network. What happens: packets (belonging to connection $A D$ ) are inserted in $\lambda_{1}$ (normally dedicated to connection AE), we call this overspilling, hence the name Overspill Routing In Optical Networks (ORION). Thus, packets inserted in a wavelength different from the lightpath of the connection are called overspill packets. Likewise, packets sent on their correct lightpath are lightpath packets. When packets of connection $A D$ are inserted in $\lambda_{1}$, node $b$ has to be able to detect these, get them out and let $B$ handle them. They have to be routed further to their destination, which, in this case, is possible by sending them out again as overspill packets on $\lambda_{3}$ (or again $\lambda_{1}$ ). At node $c$ they need to get lifted out again and be handed over to $\mathrm{C}$, which does the same. In our case they are sent out again as overspill packets, again on $\lambda_{3}$ or $\lambda_{1}$. The packet flow for connection $A D$ can be seen on Fig. 1, and is indicated by the numbers in the packets. Packets in gray travel on an ORION interface (see further) between the OXC (b) and the IP/MPLS router (B). The packets in white travel on wavelengths belonging to other connections, so capacity on links is shared!

Obviously, this kind of low-level overriding of wavelength switched behavior and wavelength breakins needs modification of the nodes. As we will show in the next sections, we can do this by adding functionality. More importantly, existing components can be re-used, while the extra components are already commercially available today! Also, it is very important to note that currently we mandate overspill packets to follow the same route as its lightpath, but on a different wavelength. In other words, when looking at the network as a point to point WDM network (i.e. the actual, physical topology), no deflection routing takes place. This is mainly to avoid the classic deflection routing problems [10]. Another design choice is that an overspill packet always gets $O E$ converted at the next encountered node (thus, it never bypasses, like a lightpath packet does). Both these design choices are mainly to clearly separate the two modes of operation in an ORION network-point to point WDM like for overspill, and wavelength switched for lightpath packets-and to avoid further complicating the design (needlessly).

Note that the overspill mechanism is meant as a temporary solution for a transient overload scenario. If the experienced imbalance in the network however remains for a longer period of time, wavelength paths should be re-arranged to accommodate for the changing traffic pattern. Typically this would mean either an intervention from the operator or a dynamic re-arrangement protocol. Thus, overspill is meant as a solution for the time frame not handled by these slower mechanisms. This would mean overspill could work in a time-frame seconds up to even hours or days, depending on the underlying re-arrangement technology and the severity of the imbalance. The overspill mechanism itself could even function to postpone unnecessary tearing down and construction of wavelength paths, thereby enhancing network stability. 


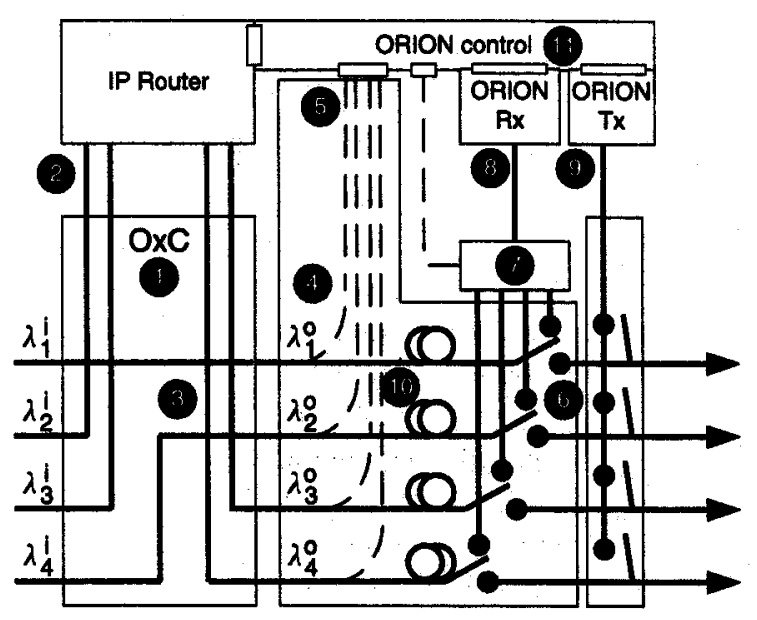

Fig. 2. Block design of an ORION node.

\section{A generic ORION node}

So how do we achieve the described scenario? Fig. 2 shows, in general, how. We need the following extra functionality:

(1) When IP packets are transported on a wavelength, we need some way to mark whether they are on their proper wavelength, or whether they are in overspill mode.

(2) This marker needs to be easily recognizable, without having to opto-electronically convert each passing packet, and with a very low processing complexity (i.e. without fully examining its header, including table look-ups etc.). This sets ORION apart from Optical Packet Switching (OPS) and Optical Burst Switching (OBS), making it a true hybrid.

(3) When the marker is detected, the overspill packet has to be extracted, but lightpath packets should be left on the wavelength. In other words, no lighttrees, drop \& continue nor broadcast \& select like scheme.

(4) The node must be able to insert overspill packets in a wavelength path passing through if capacity is available, without disturbing existing lightpath traffic on that wavelength.

In this section we assume such a marker exists. Ways to achieve the marker functionality (especially to fulfill condition (2)) are explained in Section 6.
Fig. 2 shows a complete example ORION node. First, as with a classical wavelength switched node, there is an optical cross-connect (OXC) (1), lambda $a_{j}^{i}$ denotes wavelength $j$ at the OXC input, while lambda $a_{j}^{o}$ denotes the same wavelength at the output. This OXC has four wavelengths coming in, of which two, $\lambda_{2}^{i}$ and $\lambda_{3}^{i}$ are terminated towards the electrical IP router (2). The two others, $\lambda_{1}^{i}$ and $\lambda_{4}^{i}$, pass through transparently (3). Each packet is assumed to be Intensity Modulated (IM), with the possibility to have the marker, indicating overspill status. To detect this marker, we split off some of the power at (4) and process it at (5). If the marker indicates an overspill packet, $1 \times 2$ fast optical switches (6) are set up to lead the packet towards the electrical IP router. But first, to save interface cards, we group together (7) all wavelengths. It reflects the expectation that packets in overspill will form the exception rather than occur very frequently. It however also implies that we need some contention resolution for the case that both passthrough wavelengths contain overspill packets at the same time, since we can only receive one overspill packet at a time. Deciding on how many wavelengths should be grouped together in this way is a design parameter which depends on how many overspill packets there can be expected simultaneously. In general, we reduce the range of wavelengths where $1 \times 2$ switches are installed, $M$, to a smaller range N. More on this in Section 8. The current contention resolution scheme we assume is a simple "first come first serve" mechanism. In the event of contention packets are dropped. Since overspill is a "last resort" to be used for otherwise lost low(er) priority packets, this should suffice.

After the $1 \times 2$ switch is set, the overspill packet can be received through a wide-band receiver (8). The electronic IP router, by means of the simple electronic ORION control block, can then decide (11) to either send it via the normal regime (on a lightpath originating at that IP router) with fixed (or slowly tunable) lasers, either again in overspill mode (9). In standard ORION a packet always remains in overspill mode (once overspill, always overspill). If, however, we allow overspill packets to enter a (underused) wavelength path originating at an intermediate node leading to the correct destination (for example, if $\lambda_{3}^{0}$ goes to the same end node as the detected overspill packet), we use the term ORION with 
wavelength 1 the-entry [13]. Note that there are no $1 \times 2$ swithes neoded on wavelengths $\lambda_{2}^{o}$ and $\lambda_{3}^{\circ}$, since these wavelengths are received by the IP router anyway. They can, however, be useful when rearranging the network (in the ASON way). After such a rearrangement, $\lambda_{2}^{o}$ and $\lambda_{3}^{o}$ may become part of a wavelength path, and thus no longer terminate locally, at which point the overspill capability on these wavelengths can be put to good use.

The reading and detection, together with deciding on eventual contention resolution and the setting of the $1 \times 2$ switches of course takes some time. Therefore, we provide for some delay lines (10). The required delay lines also have a second function if we want to insert packets in overspill mode on the two passthrough wavelengths. The same way we used a marker to identify overspill packets, we can also use it for detection of idle periods on the wavelengths, indicating when lightpath packets occupy the line. The delay line then provides "a window in the future" to provide time for finding a large enough gap and processing time (another example of this approach of fiber delay lines allowing for a window in the future, is described in [16]). The markers thus can have a double function, illustrated in Fig. 3:

(1) Identify overspill packets.

(2) Identify the start and end of occupied periods on the line.

The information about availability is sent to the ORION control module (11), whose function now also is to provide the electrical edge router with a wavelength on which an overspill packet can be sent, should the provided (lightpath) capacity be insufficient. To be able to access all outgoing wavelengths without having to install a transmitter (and interface card) for each wavelength, a fast tunable laser (which we term ORION transmitter) can be employed (9). Again, the shown schematic implies that only one overspill packet can be sent at a time, which can be changed by adding more tunable lasers.

The advantages of ORION are already reflected party in the node design. First of all, the logic controlling the overspill part needs to work only with the markers. Secondly, packets which are inserted on a direct wavelength path are left untouched until they reach their destination. This means that no expensive interface cards at the line tate are needed

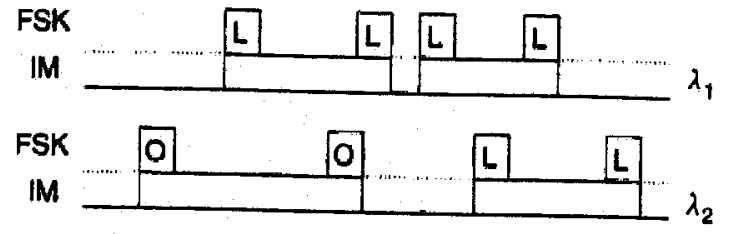

Fig. 3. The double use for the marker functionality, " $O$ " marks overspill packets while " $L$ " marks lightpath packets.

for pass-through traffic, and that the delay of the packets throughout the network can be guaranteed, and is minimal. Once they enter the direct wavelength path, their delivery is assured (apart from physical corruption). And finally, since we only rely on information obtained via the markers, this part of the node is totally oblivious to the underlying line encoding or data rate. Finally, note that the IP router (2) in this design can be replaced by an OPS/OBS switch. The advantages of ORION still apply: smaller OPS/OBS switches (less traffic seen, which means less processing) and a high utilization rate, resulting in potentially cheaper networks.

As a result of the overspill concept the wavelength resources in fact seem shared between the connections, while the default behavior is to send via the lightpaths, with a minimum processing burden for the routers. Only a small fraction of the traffic is expected to use the overspill mode, but the benefits can be quite large in terms of number of wavelengths needed in the network and number of packets to be handled, as will be shown in Sections 7 and 8.

\section{Relation to the light-trails and light-bus}

The light-trails [5] and light-bus [6] concepts are very similar to ORION in terms of design, but differ in a few fundamental ways. That is why we explicitly highlight some differences:

(1) Both light-bus and light-trails have an optical receiver and transmitter at each wavelength. In ORION, those are limited in number by the passive combiner and the tunable laser.

(2) Deciding whether or not to terminate in light-trails or light-bus is based upon processing of the optical Intensity Modulated (IM) signal, for each passing packet in the trail. ORION only relies on a marker, and only for the overspill packets. 
(3) Sensing for free space on the line is done similarly. The described ORION way of finding a gap large enough to fit a packet is very like the most advanced version of the light-bus concept. Both use FDL's to find a large enough gap for a packet waiting for transmission, avoiding garbling up existing traffic.

(4) All packets in the network are transported through light-trails and light-buses, while in ORION, most traffic is simply wavelength switched.

From a high-level viewpoint, the sensing and actual receiving in overspill in ORION is split in two functions, since the former is deemed to be a lot less complex than the latter. Furthermore, ORION has a strict priority system built in, due to direct usage of wavelength switched traffic. In light-bus or light-trails, this can be regarded as giving the source node of a light-trail or light-bus absolute priority over others. Traftic inserted by subsequent nodes in the trail corresponds to using the overspill mode in ORION. While overspill traffic has no fixed priority in ORION (from an optical viewpoint), since they are processed electronically at each hop, in light-bus and light-trail there is (traffic from upstream nodes have priority).

\section{Node implementation options}

So how do we implement this marker, which is critical for the ORION concept? In this section we detail two options, one fully electronic based and another which requires some all-optical circuitry.

\subsection{Electronic overspill, with an orthogonal signal}

The original design relied on total electronic control when dealing with the marker. The inspiration came from the IST-STOLAS project [17], where a.o. orthogonal labeling of optical signals is studied. Thus, one option would be to implement the marker as an orthogonal signal, for example a Frequency Shift Keying (FSK) signal, which would look like Fig. 3. In essence, the orthogonal signal is modulated at a much lower speed than the IM signal, which allows for cheaper electronics and easy extraction of the marker, without tampering with the actual payload of passthrough packets. Thus we control high speed data by means of a low speed signal, only processing the high speed data when necessary. Two options were considered, differing in the way detection of lightpath packets is done:

(1) An orthogonal signal implements all marker functionality, possibly with length indication: A very straightforward and simple implementation of ORION marks packets by adding a very simple orthogonal signal at the beginning and end of each packet, as in Fig. 3. The first signal marks the beginning of the packet while the second marks the end. This, however, means that we do not know beforehand how long each packet is, and that the orthogonal signal interpreter must constantly monitor the line for the stop signal. A better way of marking may be to add a length indication to the start signal, removing the need for the stop signal. This halves the amount of markers, but makes each one longer and leads to more complex electronics.

(2) Power detection for idle detection, instead of using the orthogonal signal: A further simplification is using power detection to find out where potential gaps are for inserting overspill packets. This mandates that no power is on the line when there are no packets, but it gives a big advantage: no more orthogonal signal required for lightpath packets! Only overspill packets need an orthogonal signal. This means only the fast tunable lasers (of the ORION output interface) need to be able to put an orthogonal signal on top of the IM signal, and the orthogonal signal never travels more than 1 hop (relaxing transmission requirements). Both simplify the system, which may lower costs and allow for reuse of more traditional components.

\subsection{Optical overspill, with an IM header}

All-optical routers have been a focus of research for quite some time. The design goal is to switch all packets completely optical, including all-optical header processing. While this approach may very well be the best solution in the long run, most optical designs quickly run into scalability problems, a.o: because very few labels can be supported all-optically. Therefore all-optical switches may still be a long way off. ORION however, can benefit greatiy from existing simple all-optical logic since a very limited label space 


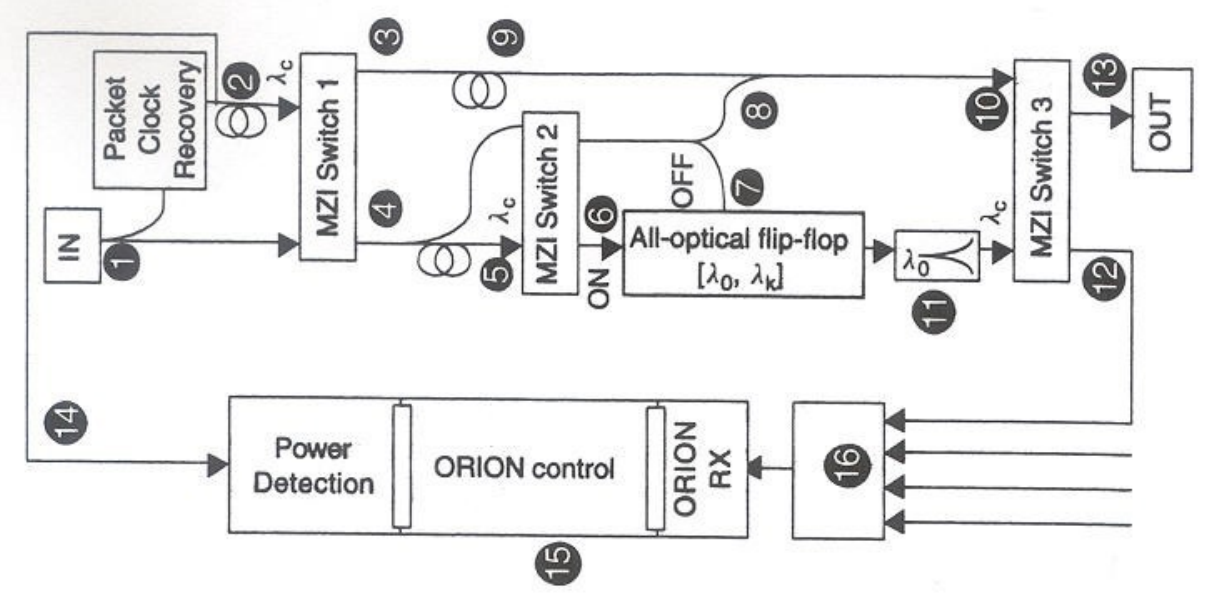

Fig. 4. Example of an all-optical overspill detection circuit.

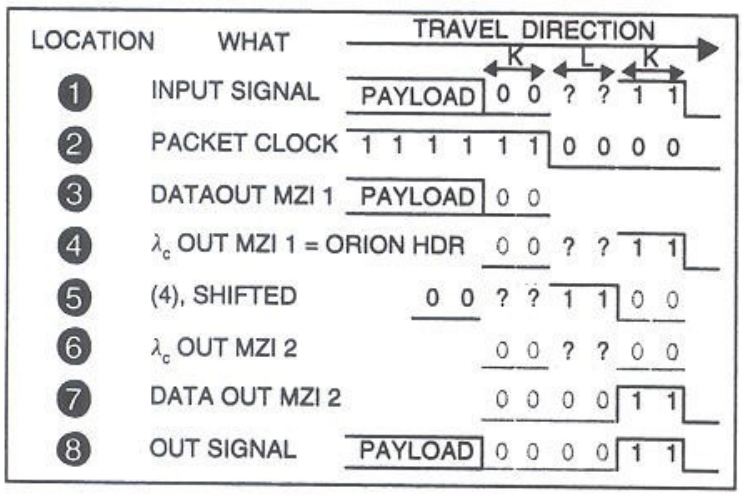

Fig. 5. Timing diagram for the all-optical circuit.

is sufficient (indication of overspill or lightpath could be possible with just a few bits). Fig. 4 shows how such a control circuit may be implemented, using demonstrated technology reported in [18] and [19]. The all-optical circuit, shown in Fig. 4, replaces the gray area in the original design of Fig. 2. Fig. 5 shows some timing aspects of the circuit in more detail. The IST-LASAGNE project [20] aims to implement and integrate similar designs for building an all-optical router.

Each packet is assumed to be Non Return to Zero (NRZ) IM modulated preceded by the following header: a $K$ bit pre-amble of $1 \mathrm{~s}, L$ bits of overspill indication (either all 0 s, or all $1 \mathrm{~s}$ ), and a $K$ bit post-amble of $0 \mathrm{~s}$. The $(2 K+L$ bits long) header, termed ORION header, is the marker, replacing the orthogonal label from the electronic implementation (Fig. 5 assumes $K$ and $L$ both 2 bits long). The packet enters the optical circuit at (1), after which its clock is extracted, using the same technique employed in [18]. This clock signal is delayed $K+L$ bits with regard to the payload (2). This allows for separation of the ORION header (4) and payload (3) all-optically in a Mach-Zehnder Interferometer (MZI) switch 1, as in [19]. The retrieved ORION header is then split at (4). The $\lambda_{c}$ arm is delayed $K$ bits with regard to the other arm, and both are fed through MZI switch 2 . The output of the "data" arm (7) of MZI switch 2 is always a pulse train of $K$ ones. These will tune the optical flip-flop to $\lambda_{0}$ with each new packet arriving, overspill or not. The output of the $\lambda_{c}$ arm (6) of MZI switch 2, depends on the $L$ bits of 0 s or 1 s (denoted "?", cf. Fig. 5). If "?" = 0, no pulse will be present. If "?" $=1$, a pulse of $L$ bits will exit on the $\lambda_{c}$ arm (6), tuning the flip-flop to $\lambda_{k}$. As the timing diagram at (6) and (7) in Fig. 5 shows, the pulse on the data arm always precedes the eventual $\lambda_{c}$ arm pulse, resulting in a tuning to $\lambda_{k}$ until the next ORION header is processed when the next packet arrives. Depending on the reaction time of the flip-flop an extra optical delay at (6) may be required. Thus, the flip-flop emits $\lambda_{0}$ on detection of a lightpath packet, $\lambda_{k}$ on detection of an overspill packet. $\lambda_{0}$ is filtered out (11), leaving the $\lambda_{k}$ signal to control MZI switch 3. This switch controls the final direction of the payload, entering at (10) after compensating for delay and flip-flop stabilization at (9). If the payload is detected as overspill it is directed 
to the ORION receiver (16), otherwise it is allowed to continue to the next node (13). At this point, the payload is correctly switched, but the ORION header is consumed. Therefore, we split the pulse of $K$ bits at (8) and insert it at $K+L$ bits in front of the payload. This rewrites the ORION header as a normal packet, allowing normal packets to continue unchanged to the next hop. If the re-insertion of the ORION header proves to be too difficult, we can also compensate for it at transmission time by adding $H$ times the ORION header in front of a normal payload, with $H$ the amount of hops to be travelled. One header is then consumed at each hop. Thus lightpath traffic travels fully transparent through the switch, while ORION traffic gets sent to the ORION receiver. Note that by rewriting the ORION header, overspill packets will be marked as a normal packet. Since they are converted to the electronic domain and arrive at a converting interface dedicated to receiving overspill packets, this does not pose a problem at all. In fact, the ORION header may be used as (part of a) pre-amble for the $\mathrm{OE}$ conversion. As discussed in Section 4 the second function of the ORION marker was detection of passthrough traffic, so that information about availability of wavelengths can be passed to ORION control (15). In this design it is replaced by power detection of the clock signal (14). The delay line needed to detect a large enough idle time, (12) on Fig. 2, can be inserted after the last switch, at (13).

\section{Evaluation at network level: a dimensioning}

With ORION, the same physical resources can act as a wavelength switched and packet switched network simultaneously. In this paragraph we show the result of a dimensioning on an example topology, to quantify the advantage ORION would bring. Only standard ORION is evaluated. For a more in-depth explanation of the methodology and more results we refer to [14] and [15].

The shown case study compares standard ORION (without wavelength path re-entry) with three other technology types. We compared them using a double metric: on one hand the amount of wavelengths needed in the network to fulfill demands, and on the other hand the amount of traffic the IP routers will have to handle. The types we compared were:
- Standard ORION: Our new proposed concept, without the lightpath re-entry option enabled.

- Point to point WDM: Sometimes referred to as fully packet switched, or link-by-link grooming. this is one of the two technology types composing ORION, used for packets in overspill mode. Since full statistical multiplexing is possible, all demands over a link can be combined, resulting in a minimal amount of required wavelengths. However, a maximum amount of packets need to be switched in the IP routers.

- Wavelength switched WDM: Also referred to as "end-to-end wavelength paths", or no grooming, this is the other technology type ORION consists of, employed for lightpath packets. Since there is no statistical multiplexing between demands having either a different source or destination node, no demands can be combined. This results in the highest amount of wavelengths needed to route them. In essence, all demands on a link are dimensioned for separately. On the other hand, since there is no transit traffic anywhere, IP routers only have to route demands originating or terminating here. Thus, a minimum amount of traffic will go through the IP routers.

- Combined: As a means to compare ORION with another hybrid architecture, we also evaluated a wavelength switched WDM network combined with a parallel point to point WDM network. Unlike ORION however, these two networks are completely separate, and cannot use each other's spare capacity. Incoming traffic at the edge of the network uses the wavelength switched WDM network if capacity on a direct wavelength path is available, otherwise it is sent over the parallel point to point WDM network. Like standard ORION (also termed "once ORION, always ORION"), once traffic enters the point to point WDM network (overspill mode in the case of ORION) it does not re-enter the wavelength switched WDM network. Note that the combined architecture can be considered a form of grooming. The CHEETAH [12] concept, although slightly different, can also be considered as a form of combined.

The topology used, shown in Fig. 6, is the reference core network topology [21], developed within the COST266 [22] and IST-LION [23] projects. It describes a hypothetical pan-European network, with 


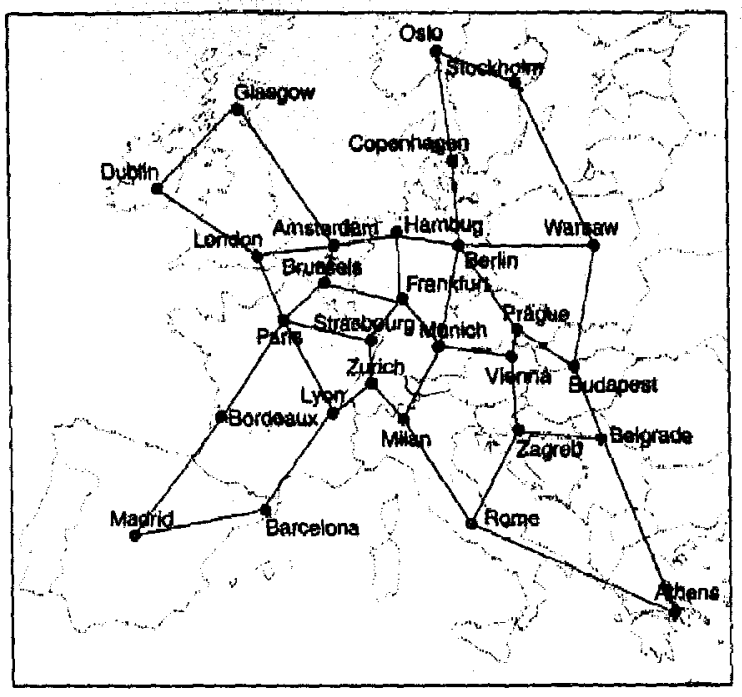

Fig. 6. Network topology used for the case study.

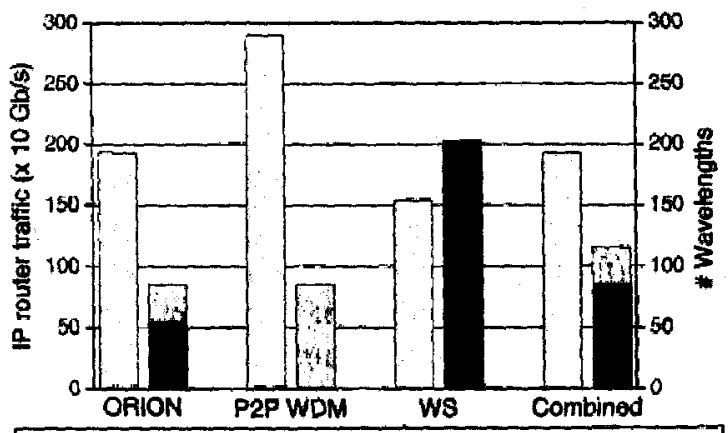

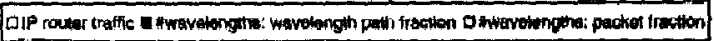

Fig. 7. Case study results, per technology type: average amount of traffic seen by $I P$ routers (left bars-left axis) and average number of wavelengths required in the network to fulfill demand (right bars-right axis).

corresponding demand forecasts for 2008 . We further assume $10 \mathrm{~Gb} / \mathrm{s}$ per wavelength installed, where each wavelength can be installed unidirectionally, and independent from others. Fig. 7 shows the results summarized. As we mentioned earlier, we look at the network dimensioned from two viewpoints: amount of packets electronically switched, and amount of wavelengths used.

When looking at average IP router load, it is very clear that point to point WDM has to handle a lot more traffic than the other three alternatives. The wavelength switched WDM solution naturally performs the best, but more interestingly, the combined technology and ORION are close to this lower limit. When looking at average number of wavelengths needed in the network, the right bars, we see the opposite (again, less is better). The wavelength switched WDM architecture uses a lot more wavelengths than the others, while ORION uses the same amount of wavelengths as the packet switched case. We also see an important feature of ORION illustrated: only a small fraction of the wavelengths are ever used for overspill (gray area). In fact, when we looked at how much traffic on average was in overspill, it was in the order of $1 \%$ of the total amount of traffic! When looking at the nodes, you can say that this performance comes at a cost: a bigger IP router has to be provisioned, but the calculated size is much closer to the wavelength switched solution than to the point to point WDM network. Combined approaches ORION in terms of wavelength usage, but uses slightly more wavelengths. Both hybrids however, are remarkably balanced in resource usage when compared to the two more extreme approaches. Therefore, it is quite clear that these types of hybrid architectures make most (economical) sense when bandwidth and switching costs are both significant cost driving factors.

Another fact, not shown on the figure, is that there are some hot spots in the network, where a lot of transit traffic passes. These hot spots result in a very large difference between the packet and wavelength switched case. For example, in Berlin, the point to point WDM solution needs to process over three times the amount of IP traffic the wavelength switched based solution sees. These hot spots also show up in other studies [1]. With ORION there is a significant improvement, even in Berlin the amount of traffic seen in the IP router is only $80 \%$ (instead of $300 \%$ ) more than for pure wavelength switched. This is due to reliance of ORION on wavelength switching as a primary transport method, which naturally benefits hot spots the most. Thus, while keeping wavelength usage minimal, IP switching demands are also reduced.

The figures presented here can vary considerably, depending on topology and traffic profile. For a more thorough study, we refer to [15]. In general though, due to the hybrid nature of ORION, ORION is the most effective when bandwidth and forwarding capacity of IP routers (whether they are optical or not) both cannot be neglected regarding cost. Thus, if one of these factors becomes totally dominant, 


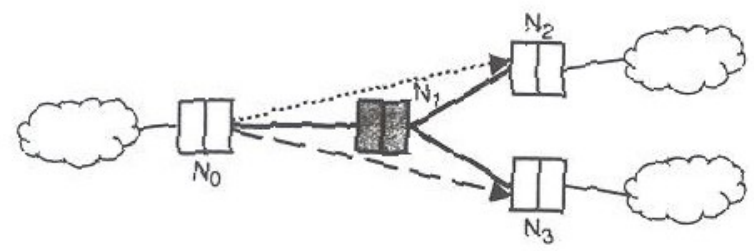

Fig. 8. Network topology used for the case study.

like bandwidth capacity was in the past, ORION will certainly not prove to be the most (cost-)effective architecture. For the immediate future however, both seem to be, and remain, major contributors.

\section{Evaluation at node level: a simulation}

In the previous paragraph we showed a case study based on dimensioning of an entire network. However, in order to keep such a dimensioning manageable, some assumptions had to be made. One of those was that the amount of overspill interfaces was always sufficient. In other words, blocking due to contention at points (7) and (9) in the ORION node was deemed non-existent. To assess a.o. how many pverspill interfaces and tunable lasers we would need to approximate such ideal conditions, we needed a finer grained approach. Hence the node simulator was built. Unfortunately, simulating an entire network scales very poorly, especially since all packet flows in the network need to be considered, which is why currently we are limited to one node in the network, and make an abstraction of the surrounding nodes. Moreover, studying such a simple case allows for more insight than we would get with an entire network study on a node level.

We discuss a simple scenario, but one which will give us some insight into ORION from a node's perspective. We depict the scenario under study in Fig. 8: a four node network. Nodes $N_{0}$ and $N_{2}$ are connected via a lightpath, on $\lambda_{1}$, while $N_{0}$ and $N_{3}$ also have a lightpath connection, on $\lambda_{2}$. This means that connection $N_{0} \rightarrow N_{2}$ and $N_{0} \rightarrow N_{3}$, can use overspill on each others' wavelengths on link $\mathrm{N}_{0}-\mathrm{N}_{2}$. We only look at what happens on this link. We consider the case where both connections $\left(N_{0} \rightarrow N_{2}\right.$ and $N_{0} \rightarrow N_{3}$ ) have a Poisson inter-arrival behavior, while an Internet mix was chosen as a packet size distribution (40 bytes, $50 \%$ occurrence, 520 bytes $37.5 \%$ occur-

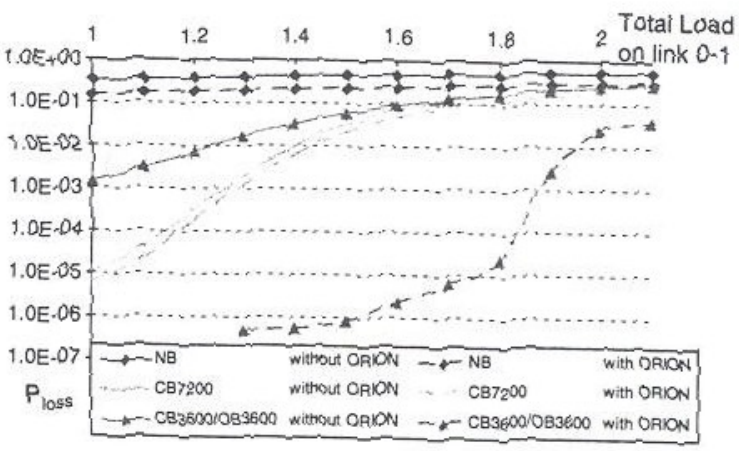

Fig. 9. Total loss (for both $0 \rightarrow 2$ and $0 \rightarrow \xi$ ) in function of total load.

rence and 1500 bytes $12.5 \%$ occurrence). The load of connection $\mathrm{N}_{D} \rightarrow \mathrm{N}_{2}$ was kept fixed at $50 \%$, while the load of connection $N_{0} \rightarrow N_{3}$ was varied between $50 \%$ and $160 \%$. Simulations are reliable: for loss higher than $10^{-4}$ the $95 \%$ confidence interval is $7.5 \%$ of the mean estimate value or lower. For low losses the confidence interval is larger, we have indicated the error bars in Fig. 10 for just one curve in order not to overload the graphs.

As a source-destination pair generates traffic according to a Poisson process, and packets have a certain (finite) length, it is possible that two packets generated in $\mathrm{N}_{0}$ and both going to the same destination node, contend for the wavelength of the lightpath. We have chosen for such a traffic generation as it mimics the aggregation of traffic that will occur in a node lìke $N_{0}$, which will be fed by some metro/access networks. Different possibilities are present here (at the sending side of $\mathrm{N}_{0}$ for our case study):

- No Buffering (NB): When a packet of a connection finds its lightpath occupied, it cannot use this wavelength, and immediately has to try going into overspill on an altemative wavelength. When this wavelength is not available, the packet is not sent out, thus is lost at the generating node.

- Circuit Buffering (CB): When a packet of a comnection finds its lightpath occupied, it can retry for a time $T_{\text {circujit }}$. When this time is over and the packet is still not sent out, it can try to go in overspill on an alternative wavelength. When such a wavelength is not available at that time, the packet is not sent out, thus is lost at the generating node.

- Overspill Buffering $(\mathrm{OB})$ : When a packet of a connection finds its lightpath occunied, it cannot use 

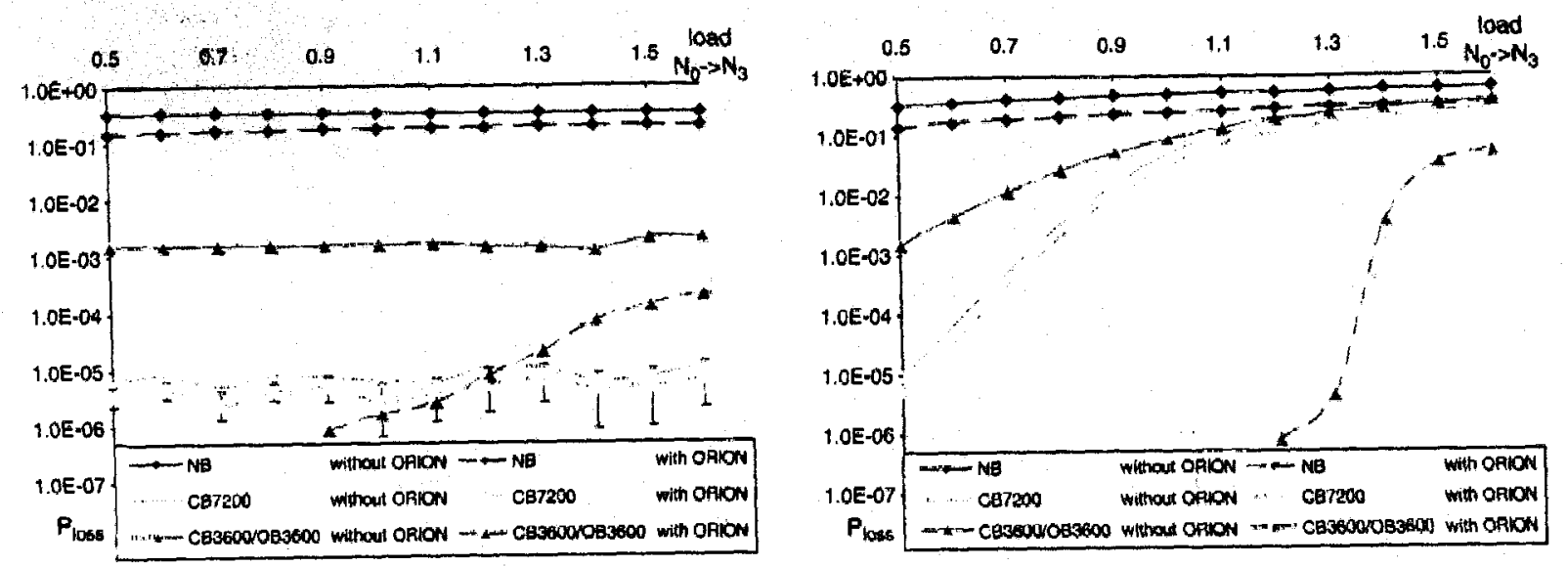

Fig. 10. Loss curves for the individual connections $N_{0} \rightarrow N_{2}$ (left) and $N_{0} \rightarrow N_{3}$ (right).

this wavelength, and immediately has to try going into overspill on an alternative wavelength. The packet can try using the overspill wavelength(s) for a specified time $T_{\text {overspill. When no overspill wave- }}$ length is available within this time, the packet is not sent out, thus is lost at the generating node. When no Overspill is installed on the network, the overspill buffer is of no use and packets simply get dropped, when their lightpath is occupied.

- Circuit Buffering and Overspill Buffering (CBOB): When a packet of a connection finds its lightpath occupied, it can retry for a time $T_{\text {circuit. }}$. When this time is over and the packet is still not sent out, it can try to go in overspill on an alternative wavelength and try this for a time $T_{\text {overspill. When no overspill }}$ wavelength is available within this time, the packet is not sent out, thus is lost at the generating node. When no Overspill is installed on the network, the overspill buffer is of no use and packets simply get dropped, when their lightpath is occupied.

We indicate the retry times $T_{\text {circuit }}$ and $T_{\text {overspill }}$ directly after the buffer type, e.g. CB7200, means a Circuit Buffer with $T_{\text {circuit }}=7200$ ns. By using a time-limited mechanism we do not put forward an a priori limit on the actual buffer size. However, by monitoring this buffer size we will be able determine a suitable one (cf. later). Note that the discussed buffers are in the electronic domain. We will look at different buffer strategies as described in the above paragraph, with the overspill option either enabled or disabled. In the case ORION is enabled the traces will be marked with ORION, when ORION is disabled we will use without ORION. The latter case is in fact operating the network purely as a wavelength switched network.

Fig. 9 shows the total loss in function of the total load on link $\mathrm{N}_{0}-\mathrm{N}_{1}$. Without buffering we clearly see a very high loss rate, due to frequent bursts of traffic overlapping in time, of which only one can use the lightpath wavelength, while others have to immediately resort to ORION. However, by using a Circuit Buffer with a retry time of only $7200 \mathrm{~ns}$, loss is greatly reduced. Enabling ORION does not have a large effect on the traffic that gets sent (i.e. not lost) in the $\mathrm{CB} 7200$ case. Using the same total retry time, but splitting this into both Circuit and Overspill Buffer (CB3600/OB3600), shows the power of Overspill: Drastically lower loss ratios are observed.

This is even clearer in Fig. 10, where we show the loss for the two connections separately. Although the connection $\mathrm{N}_{0} \rightarrow \mathrm{N}_{3}$ has only one wavelength available, it can send a load higher than one wavelength $(=100 \%)$, using the wavelength of connection $N_{0} \rightarrow N_{2}$, which is only loaded at $50 \%$. We indeed see that connection $\mathrm{N}_{0} \rightarrow \mathrm{N}_{3}$ can send more than $100 \%$ of a wavelength capacity. When we put a limit on loss of $10^{-4}$, using only a Circuit Buffer of $7200 \mathrm{~ns}$, slightly less than $70 \%$ load can be achieved. However in the CB $3600 / 0 B 3600$ a load of 1.45 can be handled. This is because connection $\mathrm{N}_{0} \rightarrow \mathrm{N}_{3}$ can now use the spare wavelength of $0 \rightarrow 2$ for its overload, exactly what ORION was conceived for! Note that for the traffic to be able to end up in $\mathrm{N}_{3}$, link $\mathrm{N}_{1}-\mathrm{N}_{3}$ also needs to carry a wavelength that is not fully used for its connection. 

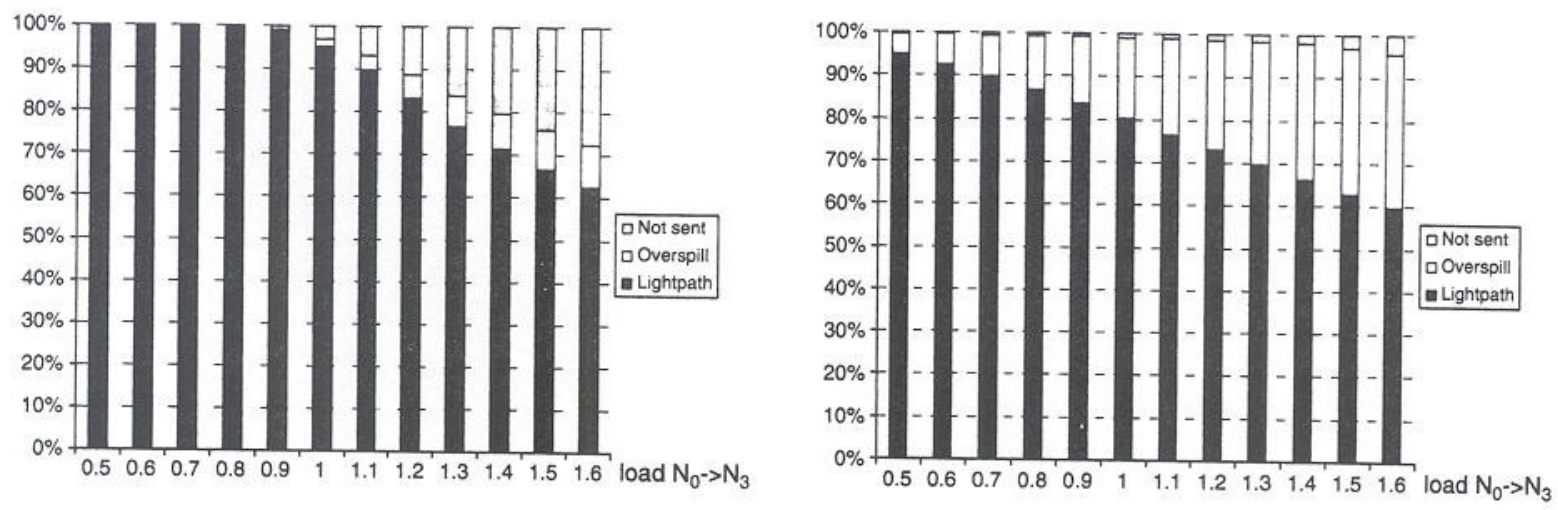

Fig. 11. Connection $\mathrm{N}_{0} \rightarrow \mathrm{N}_{3}$ traffic statistics for CB7200 (left) and CB3600/OB3600 (right).

This could be any connection in the network routed ver link $\mathrm{N}_{1}-\mathrm{N}_{3}$. The unstable nature of the CB7200 urves for connection $\mathrm{N}_{0} \rightarrow \mathrm{N}_{2}$ is due to the low oss rate, as only few packets get lost, simulation time vould be unreasonably high to get stable results.

Fig. 11 shows how traffic of connection $\mathrm{N}_{0} \rightarrow \mathrm{N}_{3}$ s sent. With a Circuit Buffer of $7200 \mathrm{~ns}$ we see that Imost all traffic is sent out via the lightpath. When raffic goes in overload (load $>1$ ), we see a fast raise $\mathrm{n}$ the amount of traffic that cannot be sent out. In he case CB3600/OB3600, ORION kicks in and can tandle a traffic load over $100 \%$. Also note that even Iefore $100 \%$ the amount of traffic sent in overspill node is significant. Only for very high loads we see hat packets start to get lost, corresponding to the steep ise in the loss curve of Fig. 9.

We also show the occupation of the Circuit nd Overspill Buffer in the case CB3600/OB3600 n Fig. 12. We see the circuit buffer can be kept ather small: about $30 \mathrm{~KB}$ is sufficient. The memory equirement has a steep rise as the load becomes high, $\mathrm{s}$ traffic is kept long before it can (possibly) access an verspill wavelength. With a limitation of $1 \mathrm{MB}$, we ould however still get slightly less than $140 \%$ of load in connection $\mathrm{N}_{0} \rightarrow \mathrm{N}_{3}$. This closely corresponds to ae value we found with the loss limitation of $10^{-4}$.

\section{'. Concluding remarks}

In this paper we presented the ORION concept and iscussed several aspects, ranging from the concept self over various implementation options through

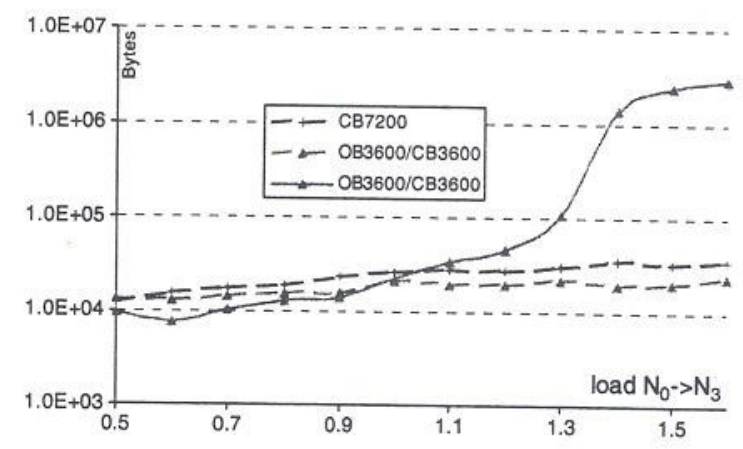

Fig. 12. Maximum buffer usage for connection $\mathrm{N}_{0} \rightarrow \mathrm{N}_{3}$ for Circuit Buffer (dashed) and Overspill Buffer (full).

an example of the evaluations we performed in the past. The most important feature of ORION itself is that it acts like a wavelength switched network, but gives the unused capacity free in the form of a point to point WDM network. The unused capacity is then consumed through the overspill mechanism, resulting in a true hybrid design. The shown case studies and others [14,15] show that these potential gains can be significant indeed. Controlling such a hybrid may look difficult, but in [13] we already show ORION to be compatible with the GMPLS framework, as well as ORION with re-entry option (where overspill packets can enter a lightpath at intermediate nodes). Another re-occurring question relates to out-of-order delivery, since overspill packets will experience a higher delay than packets going through the normal wavelength paths. This essentially is a more general problem: given multiple paths for a demand, how to distribute traffic. This is not an 
ORIOW 14 fe problem, and in fact is tackled (and standarditad) in existing common technologies like in ethernet link bundling (e.g. IEEE 802.3ad). The solution basically would amount to remapping flows (or bundles of flows) in and out of overspill, instead of single packets at a time. If the flow resolution is fine enough, this should be sufficient to avoid most reordering situations. A testbed is currently being built to evaluate this kind of remapping algorithms and the impact on live traffic streams. Regarding future work the main paths currently explored are more realistic single node simulation scenarios taking into account overspill contention (based on the already performed dimensioning work) and the evaluation of the additional value of the re-entry option. With the knowledge gained from the former we will e.g. be able to assess how many overspill interfaces and transmitters we would need and what a realistic fill ratio may be for various kinds of traffic. This will then guide the design of the size and structure of the "ORION concentrator", which is the grouping module used to reduce $\mathrm{O} / \mathrm{E}$ interfaces. The study of the reentry option on the other hand will give an indication on whether or not the performance gain will be worth the increased control cumplexity. In any case, with the results we obtained so far, ORION seems to be the best answer to the electronic bottleneck to date. While most answers usually try stretching electronics beyond their ultimate limits, or simply throw more bandwidth at the problem, ORION simply takes the best from both worlds. Electronics for processing at acceptable speeds, optics for bulk high-transfer and bandwidth.

\section{Acknowledgements}

This work was partly funded by the European commission through their Information Society Technologies (IST) program, more specifically through the projects STOLAS (IST-2000-28557), LASAGNE (FP6 506760), and NOBEL (FP6 507509). The Flemish government also funded a part of this research through the IWT-GBOU project "Optical Networking and Node Architectures". E. Van Breusegem and D. Colle thank the "Institute for the Promotion of Innovation through Science and Technology in Flanders" (IWT-Vlaanderen) for its financial support for their respective Ph.D. and postdoctoral grants.
J. Cheyns is Research Assistant with the Flemish Fund for Scientific Research (FWO).

\section{References}

[1] S. De Maesschalck et al., Pan-European optical transport networks: an availability-based comparison. Photonic Network Comm. 5 (3) (2003) 203-225.

[2] P. Green, Progress in optical networking, IEEE Commun. Mag. 39 (2001) 54-61.

[3] S. Bjornstad, D.R. Hjelme, N. Stol, A highly efficient optical packet switching node design supporting guaranteed service, in: Proceedings of ECOC 2003, Mo4.4.5, Rimini, Italy, September 2003, pp. 110-111.

[4] S. Bjomstad, M. Nord, D.R. Hjelme, QOS differentiation and header/payload separation in optical packet switching using polarisation multiplexing, in: Proceedings of ECOC 2003 . Mo3.4.6, Rimini, Italy, September 2003, pp. 28-29.

[5] A. Gumaste, I. Chlantac, Light-trails: an optical solution for IP transport, J. Opt. Nerw, 3 (2004) 261-281: http://www.osa-jon.org/abstract.cfm?URI=JON-3-5-261,

[6] S. Balasubramanian, Alumed Kamal, A.K Somani, Medium access control protocols for light trail and light bus networks, in: Proceedings of 8th IFIP Working Conference on Optical Network Design and Modeling, ONDM, 2-4 February 2004, Ghent, Belgium.

[7] P. Gambini al., Transparent optical packet switching: network architecture and demonstrators in tho KEOPS project, IEEE J. Sel. Arcas Commun. 16 (7) (1998) 1245-1259.

[8] C. Qiao, M. Yoo, Optical burst switching -a new paradigm for an optical internet, J. High Speed Netw. 8 (1) (2000) 36-44 (special issue on Optical Netwotking).

[9] M. Duescr, P. Bayvel, Analysis of a dynamically wavelengthrouted optical burst switched network architecture, J. Lightwave Technol. 20 (4) (2002) 574-586.

[10] F. Borgonovo, Deflection Routing, Routing in Communications Networks, Prentice Hall International (UK), Hertfordshire. UK, 1995.

[11] L.H. Sahasrabuddhe, B. Mukherje, Light-trees: optical multicasting for improved performance in wavelength-routed networks, IEEE Commun. 37 (2) (1999) 67-73.

[12] M. Veeraraghavan et al., CHEETAH: circuit-switched highspeed end-to-end transport architecture, in: Proceedings of OptiComm 2003. Dallas, TX, USA, October 2003, Pp. 214-225.

[13] J. Cheyns, E. Van Breusegem, D. Colle, D. De Winter, M. Pickavet, P. Demeester, Controlling LSPs in an ORION netwotk in: Broadnets 2004, San Jose, CA, USA, 25-29 October, 2004.

[14] E. Van Breusegem, J. Cheyns, D. Coile, M. Pickavet, P. Demeester, Overspill routing in optical networks: a new architecture for futureproof IP over WDM networks, in: Opticomm 2003, Dallas, TX, 13-17 October. 2003.

[15] E. Van Breusegem, J. Cheyns, D. Colle, M. Pickavet, P. Demeester, The effect of increased traffic variability and wavelength capacities on ORION, in: HSNMC 2004, 
30 June-3 July 2004, Toulouse, France (Proceedings also to be published in LNCS Series of Springer-Verlag).

[16] J. Cheyns, E. Van Breusegem, A. Ackaert, M. Pickavet, P. Demeester, Scheduling window for AWG based blocking optical switches, Electron. Lett. 39 (6) (2003) 546-547.

[17] K. Vlachos et al., An optical IM/FSK coding technique for the implementation of a label-controlled arrayed waveguide packet router, J. Lightwave Technol. 21 (11) (2003) 2617-2628.

[18] C. Bintjas, K. Yiannopoulos, N. Pleros, G. Theophilopoulos, M. Kalyvas, H. Avramopoulos, G. Guekos, Clock recovery circuit for optical packets, IEEE Photonics. Technol. Lett. 14 (2002) 1363-1365.

[19] C. Bintjas, K. Yiannopoulos, N. Pleros, G. Theophilopoulos, M. Kalyvas, H. Avramopoulos, G. Guekos, All-optical packet address and payload separation, IEEE Photonics. Technol. Lett. 14 (2002) 1728-1730.
[20] J.M. Martinez, D. Colle, F. Ramos, R. Van Caenegem, M. Pickavet, J. Marti, LASAGNE: all-optical label swapping employing optical logic gates in network nodes, NOC 2004.

[21] Online available at: http://ibcn.intec.ugent.be/css_design/ research/projects/IST_FPS/NRS/index.html.

[22] R. Inkret, A. Kuchar, B. Mikac, Advanced Infrastructure for Photonic Networks (extended final report of COST Action 266), Faculty of Electrical Engineering and Computing, University of Zagreb, Zagreb, Croatia, 2003.

[23] B. Puype, Q. Yan, D. Colle, S. De Maesschalck, I. Lievens, M. Pickavet, P. Demeester, Multi-layer traffic engineering in data-centric optical networks-illustration of concepts and benefits (IST LION), in: Proc. of ONDM 2003, The 7th IFIP Working Conference on Optical Network Design \& Modelling, 3-5 February 2003, Budapest, Hungary, vol. 1, 2003, pp. 211-226. 


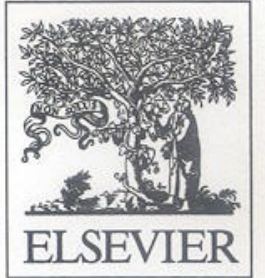

\section{Optical}

Switching and

Networking 